\title{
Research on the Complexity Mechanism of Decoy Strategies Based on Multiagent Simulation
}

\author{
Zhen Li $\mathbb{D}^{\text {, }}$, Xiaoyu Bao, Qingfeng Meng $\mathbb{D}^{\mathbb{D}}$, and Pengqun Shen \\ School of Management, Jiangsu University, Zhenjiang 212013, China \\ Correspondence should be addressed to Qingfeng Meng; mqf@ujs.edu.cn
}

Received 14 June 2020; Revised 27 July 2020; Accepted 5 August 2020; Published 30 September 2020

Academic Editor: Dimitri Volchenkov

Copyright ( $) 2020$ Zhen Li et al. This is an open access article distributed under the Creative Commons Attribution License, which permits unrestricted use, distribution, and reproduction in any medium, provided the original work is properly cited.

\begin{abstract}
Competition and diffusion of products are of great significance to companies. In this study, various true decoy strategies are constructed using different combinations of price and quality. This paper then analyzes the performance of strategies using different product competition and diffusion scenarios. The influences of neighbor nodes, reconnection probability, and herd mentality on decoy effects are explored. The results show that setting an appropriate true decoy can enhance the competitiveness of a target product to some extent. Conversely, an inappropriate decoy strategy will play a negative role, encroaching on market share. In terms of effects, changes in neighbor nodes, reconnection probability, and herd mentality will not influence the direction of the evolution of a product for the same true decoy strategies. However, the speed of evolution will be affected. These findings provide a theoretical basis for enterprises taking action to enhance product competitiveness in the market.
\end{abstract}

\section{Introduction}

People often find that decisions can be difficult to make when a choice alternative they are considering is superior to a second one in some respects but not others. However, the addition of an appropriate third product option will change consumers' choices, even make them predictable. This phenomenon is called the "decoy effect," and it is common not only in commercial products but also in gambling, employment, political candidates, and so on.

At present, a variety of research has been conducted on the decoy strategy. In verifying the effectiveness and practical applicability of the decoy strategy, Huber et al., [1] used the strategy to achieve success in beer sales, and Pan and Lehmann [2] used it successfully in bundle sales; Health and Chatterjee [3] verified the validity and practical applicability of the phantom decoy strategy based on a meta-analysis of empirical data from previous literature.

Meanwhile, some scholars focus on exploring the causes of the decoy effect and constructing a variety of theoretical explanations. For example, "Range-Frequency Theory" by Dan and Wallsten [4] found that a decoy can change a consumer's understanding of the importance of certain attributes of a product. That is, the decoy can make consumers pay much attention to the dominant dimension of the target product, thereby increasing the weight of this dominant attribute at the time of decision-making. Additionally, some scholars explain the decoy effect based on loss aversion in prospect theory. Prospect theory believes that decision-making behavior generally has two characteristics: reference dependence and loss aversion. The former postulates that utility is evaluated by comparing to a reference point and the latter states that people weigh losses more heavily than wins. Based on the theory of loss aversion, the addition of a decoy will cause the mean of all product attribute values to become a new reference point. As such, the target product could become more competitive due to consumer loss aversion [5]. In addition, some scholars have explained the decoy effect based on a heuristic-based approach, positing that adding appropriate decoy products makes decision-making easier for consumers and helps them make choices more quickly. For instance, the introduction of a nondominate decoy can increase the choice share of a target by making it appear to be a "compromise" between 
extreme options [6]. This is because the risk of choosing a compromise option is small, and the compromise option is not the worst in any attribute dimension of the product.

Factors influencing the decoy effect are also explored by scholars. Pettibone [7] studied the effect of time pressure on the decoy phenomenon and found that the longer consumers have to consider, the smaller the time pressure is and the more obvious the effect of the decoy strategy. Additionally, Connolly et al., [8] studied the effects of regret salience and accountability on the decoy effect and found that the greater the degree of regret salience and accountability, the smaller the effect of the decoy strategy. Lichters et al. [9] found that if consumers consider the economic consequences of real life when making choices, the effect of the decoy strategy will be weakened; Trueblood and Pettibone [10] believed that the decoy effect happens when consumers' preferences change, up to a certain degree, after the addition of the decoy.

In summary, the current research on the decoy strategy mainly focuses on the existence, mechanism, and influencing factors of the decoy effect. Most studies focus on the phantom decoy (i.e., when decoy products cannot be obtained by consumers in the market) while true decoys (i.e., decoy products can be obtained by consumers in the market) are less studied. Generally speaking, compared to the phantom decoy strategy, the true decoy strategy is much more complex because a true decoy can be bought in the market and occupies part of the market share. However, the availability of true decoys makes them more meaningful as there is no suspicion that they are improper phantom decoy strategies designed to deceive consumers.

At present, the research on true decoy strategy primarily focuses on two obvious tactics: the compromise decoy (i.e., the price and quality of the decoy product are higher than the target product) [10] and the asymmetrically dominated decoy (i.e., the price of the decoy product is higher than the target product and the quality is lower than the target product) [1]. However, less attention is paid to the effects of other decoy strategies on the target product and the evolution of the target product. In addition, most studies on the decoy strategy are based on individual consumers as experimental samples, and the results are obtained by observing the individual decisions of a large number of isolated consumers. However, the influence of interaction between consumers on the effect of decoy strategies has been neglected in these studies. Moreover, less attention is paid to the long-term evolution of the decoy effect when consumers interact with one another after the decoy is put on the market.

It is well known that interaction between consumers has become one of the most important factors influencing individuals' purchasing decisions. In addition to being influenced by many attributes of the product itself, such as price and quality, consumer purchasing decisions are also affected by other actors in their social network [11]. Friends, family members, and other consumers who expect to buy or have used the same product will affect our purchasing behavior. A dynamic network that evolves over time is formed based on interactions between consumers via word of mouth, recommendation, and imitation. According to the social network theory, consumers' purchasing behavior is "embedded" in the network. The structure of the network and the interaction between the individuals will influence or restrict purchasing behavior $[12,13]$. Consumer herd behavior, which is based on network interaction, is an example of this. Consumer purchasing decisions will be greatly affected by the herd mentality [14], and this phenomenon of consumer interaction is particularly obvious during Alibaba's online shopping carnival [15].

According to the analysis above, if we expect to develop a better decoy strategy and improve the competitiveness of a target product, the influence of the interaction between consumers cannot be ignored. Therefore, based on the full consideration of interactions between consumers, this paper studies the implementation of a true decoy strategy and the subsequent evolution of the decoy effect in different scenarios. These findings will provide a theoretical basis for enterprises to take measures to improve the competitiveness of target products.

This study builds upon previous research and introduces the following innovations and extensions: first, the utility of individual consumer purchase decision-making is analyzed mathematically and a feasible decoy strategy area has been proposed. Second, fully considering individuals' heterogeneity and interactions, a consumer network has been constructed and the influence of factors (neighbors nodes, reconnection probability, and herd mentality) on the development of decoy strategies has been explored. Finally, compared with the existing research, which pays attention only to the effect of true decoy strategies in a single cycle, a visual analysis of the process of competition and diffusion of different products is made in this paper.

\section{Agent-Based Model}

2.1. Manufacturer Agents. There are two manufacturers, $g_{1}$ and $g_{2}$, competing in the market, producing and selling products, $a$ and $b$, respectively. The price of product $a$ is $p_{a}$, and the quality is $q_{a}$; the price of product $b$ is $p_{b}$, and the quality is $q_{b}$. Products compete based on the two-dimensional product attributes of price and quality and satisfy $p_{a}>p_{b}, q_{a}>q_{b}$. In order to increase the sales and market share of product $a$, the enterprise plans to introduce decoy product $c$. The price of the decoy product is $p_{c}$, and the quality is $q_{c}$. Product $c$ is a true decoy that consumers can absolutely obtain in the market.

\subsection{Consumer Agents}

2.2.1. Consumer Utility and Purchase Decisions. The model contains two manufacturers and $N$ consumers. $U_{i}^{j}$ represents the utility that product $i$ brings to consumer $j(j \in(1, \ldots, N))$, which indicates the motivation and willingness of consumer $j$ to purchase the product. Consumer $j$ makes the purchasing decision according to the utility of the product. According to the literature [16], $U_{i}^{j}$ is shown as the following formula: 


$$
U_{i}^{j}=\mu_{i}^{j} * p_{i}+\rho_{i}^{j} * q_{i} .
$$

Utility $U_{i}^{j}$ is influenced by two main factors. One is the price of product $i . \mu_{i}^{j}$ in the previous formula indicates the price sensitivity parameter of consumer $j$ for product $i$. As we all know, consumers' motivation to choose a product is affected by its price. Price sensitivity, a characteristic of consumers, indicates the perception of the practical and emotional value of products, and different people have different price sensitivities. According to the price sensitivity distribution model [15], the lower the price of a brand, the lower the price sensitivity caused by the brand. In other words, this brand will present little barrier to a consumer's motivation to buy. The price sensitivity of a consumer is the power function of the difference between the actual price, $p_{i}$, and the expected price, $p_{e}$, of this good, which is a negative number, as shown in the following formula:

$$
\mu_{i}^{j}=-\partial_{j}^{\left(p_{i}-p_{e}\right)}+\kappa_{j},
$$

where $\partial_{j}>1$ and $\kappa_{j}$ is a constant. $\kappa_{j}$ depends mainly on consumer $j$ 's socioeconomic attributes, $\mathrm{SE}_{j}$. For example, the socioeconomic attributes of entrepreneurs and the unemployed are quite different, and the price sensitivity of rich people is lower than that of the poor or the unemployed. That is to say, $\kappa_{j}$ is inversely proportional to socioeconomic attributes, which means $\kappa_{j}=1-\left(\mathrm{SE}_{j} / \mathrm{SE}_{\max }\right) . \mathrm{SE}_{\max }$ is the maximum socioeconomic attribute of consumers. In addition, the expected price of the product is usually replaced by the average price of similar products, as shown in equation (3). This article mainly refers to the application in Zhang and Zhang [16] when constructing the model of the consumer decoy effect. $p_{e}$ is the consumer's expected price of this type of product. $p_{e}$ is difficult to derive and can be substituted with the average price of the product, representing the average price of $m$ products in the market:

$$
p_{e}=\frac{\sum_{i=1}^{m} p_{i}}{m} .
$$

On the other hand, $\rho_{i}^{j}$ in equation (1) indicates the quality sensitivity of consumer $j$ on product $i$. The psychological theory of consumer outlier avoidance suggests that when a consumer chooses a brand, the more the brand approximates the consumer's expected quality for this kind of product, the more sensitive the consumer is to the quality of the brand. The $\rho_{i}^{j}$ expression is shown in the following formula:

$$
\rho_{i}^{j}=\beta_{j}^{\left|q_{i}-q_{e}\right|}+\varepsilon_{j} .
$$

$\beta_{j}$ meets $0<\beta_{j}<1$, and $\varepsilon_{j}$ is influenced by the socioeconomic attribute $\mathrm{SE}_{j}$. For example, consumers with higher incomes pay much attention to quality when they make purchasing decisions. In this situation, $\varepsilon_{j}$ is proportional to $\mathrm{SE}_{j}$; that is, $\varepsilon_{j}=\left(\mathrm{SE}_{j} / \mathrm{SE}_{\max }\right) ; q_{e}$ indicates consumer expectations of quality, the average of similar products, as shown in the following formula:

$$
q_{e}=\frac{\sum_{i=1}^{m} q_{i}}{m} .
$$

Based on formulas (1) to (5), the utility of consumer $j$ can be further expressed as the following formula:

$$
U_{i}^{j}=\left(-\partial_{j}^{\left(p_{i}-p_{e}\right)}+\kappa_{j}\right) * p_{i}+\left(\beta_{j}^{\left|q_{i}-q_{e}\right|}+\varepsilon_{j}\right) * q_{i} .
$$

Consumer $j$ makes purchasing decisions based on the utility of product $i$; that is, consumer $j$ will choose the maximum utility among $U_{a}^{j}, U_{b}^{j}$, and $U_{c}^{j}$.

2.2.2. Consumer Herd Psychology and Interactive Networks. In real life, consumer purchasing decisions are affected by the price and quality of products. These decisions are also influenced by other consumers through recommendations, word of mouth, and so forth. In addition, in the purchasing decision process, there will be a certain consumer herd mentality $[17,18]$. In the interaction between consumer $j$ and other consumers, the price sensitivity, $\partial_{j}$, and quality sensitivity, $\beta_{j}$, can be changed due to herd mentality. Parameter $\theta_{j}$ indicates the strength, the influence of consumer $j$ 's herd in terms of the product price and quality, and is subject to normal distribution $[0,1]$. The closer $\theta_{j}$ is to 0 , the less likely the consumer is to be influenced by others, the weaker the effect of the herd is, and the closer it is to 1 , the more likely he or she is to be influenced by others. The rules of the changes of consumer $j$ 's price sensitivity, $\partial_{j}$, and quality sensitivity, $\beta_{j}$, are shown in the following two formulas, respectively:

$$
\begin{aligned}
& \partial_{j}^{t+1}=\left(1-\theta_{j}\right) \partial_{j}^{t}+\theta_{j} \partial_{\mathrm{je}}^{t}, \\
& \beta_{j}^{t+1}=\left(1-\theta_{j}\right) \beta_{j}^{t}+\theta_{j} \beta_{\mathrm{je}}^{t},
\end{aligned}
$$

where $t$ indicates the cycle of the consumers' purchasing. The price sensitivity of consumer $j$ in the $(t+1)$ cycle is affected by the two aspects of the previous cycle (the cycle of $t$ ). On the one hand, it is influenced by the consumer's own price sensitivity, $\partial_{j}^{t}$, which is an attribute of the individual consumer. On the other hand, it is also affected by the interaction with other consumers, that is, $\theta_{j} \partial_{j e}^{t}$. $\partial_{j e}^{t}$ is the mean of price sensitivity of consumers who interact with consumer $j$, indicated as $\partial_{j e}^{t}=\sum_{l=1}^{h} \partial_{j l}^{t} / h$, which means that the number of consumer $j$ s s neighbors is $h$ in the cycle of $t$, and the price sensitivity coefficient of the neighbors is indicated as $\partial_{j l}^{t}$, where $l \in(1, \ldots, h)$. The price sensitivity of consumer $j$ at period $t+1$ can be calculated from the weighted summation of the above two parts. $\theta_{j}$ represents the degree of consumer $j$ herd behavior. Similarly, $\beta_{j e}^{t}$ represents the mean coefficient of consumer $j$ 's quality sensitivity, as influenced by his or her neighbors, in period $t$, which is indicated as $\beta_{\mathrm{je}}^{t}=\sum_{l=1}^{h} \beta_{\mathrm{j} 1}^{t} / h$.

Previous research has shown that the consumer interactive network has typical small-world network characteristics $[19,20]$, and this paper depicts the interactive network between consumers based on a small-world network. The 
small-world network contains $N$ nodes based on the specific rules of loop networks. Each node is connected to its nearest neighbor through $K$ node to $K$ edge, and it is assumed that $N \geq K \geq \ln N \geq 1$ when each edge of an endpoint remains the same. $P_{r}$ indicates the probability of another endpoint connection; by adjusting the $P_{r}$ value, either a network can be completed completely according to the rules $\left(P_{r}=0\right)$ or it can transition to a completely random network $\left(P_{r}=1\right)$. Therefore, consumer $j$ 's purchasing decision will be affected by the characteristics of the small-world network, such as the number of nodes $K$ and reconnection probability $P_{r}$. The number of nodes can be represented by the number of other consumers who interact with consumer $j$ in the purchasing decision process. The reconnection probability can represent the influence other consumers have on consumer $j$ in each purchase decision. To sum up, consumers will change their utility evaluation under the influence of their own preferences and herd effects. The utility function for evaluating different products is shown in the following formula:

$$
U_{i}^{j}(t+1)=\left\{-\left[\left(1-\theta_{j}\right) \partial_{j}^{t}+\theta_{j} \partial_{j e}^{t}\right]^{\left(p_{i}-p_{e}\right)}+\kappa_{j}\right\} * p_{i}+\left\{\left[\left(1-\lambda_{j}\right) \beta_{j}^{t}+\lambda_{j} \beta_{j e}^{t}\right]^{\left|q_{i}-q_{e}\right|}+\varepsilon_{j}\right\} * q_{i} .
$$

\section{Theoretical Analysis of the Feasible Area for the Decoy Strategies}

It is assumed that a consumer first chooses a product, $b$, but he or she perceives little difference in utility between product $a$ and product $b$. That is to say, $U_{b}^{j}-U_{a}^{j}=e$, where $e$ is a parameter close to 0 , although $U_{b}^{j}>U_{a}^{j}$. The smaller the value of $e$, the more easily consumer $j$ is affected by the decoy. We assume that $E_{1}$ is the mean of the price and quality of products $a$ and $b$ without the decoy, and its location is $E_{1}\left(\bar{q}_{1}, \bar{p}_{1}\right)$, where $\bar{q}_{1}=\left(q_{a}+q_{b}\right) / 2$ and $\bar{p}_{1}=\left(p_{a}+p_{b}\right) / 2 . E_{2}$ is the new mean of the price and quality of all the products in the market, and its value is $E_{2}\left(\bar{q}_{2}, \bar{p}_{2}\right)$, where $\bar{q}_{2}=\left(q_{a}+q_{b}+\right.$ $\left.q_{c}\right) / 3$ and $\bar{p}_{2}=\left(p_{a}+p_{b}+p_{c}\right) / 3$. In a scenario with no decoy, $d_{p a}$ represents the difference between the price of product $a$ and the average price of the product category (products $a$ and $b$ ) and $d_{q a}$ indicates the difference between the quality of product $a$ and the average quality of the product category (products $a$ and $b$ ). In the same way, when decoy $c$ is added, $d_{q a}^{\prime}$ and $d_{p a}^{\prime}$ denote the new differences between the price of product $a$ and the average piece of the new product category (products $a$ and $b$ and decoy $c$ ) and between the quality of product $b$ and the average quality of the new product category (products $a$ and $b$ and decoy $c$ ), respectively. The meaning of the parameters of product $b$ is similar to that of product $a$. The equations are listed in Table 1 .

$U_{a}^{j}$ and $U_{b}^{j}$ denote the consumer's motivation to purchase products $a$ and $b$ when there is no decoy product $c . U_{a}^{j^{\prime}}$ and $U_{b}^{j^{\prime}}$ denote the consumer's motivation to buy products $a$ and $b$ when decoy product $c$ exists in the market. Therefore, the formulas for $U_{a}^{j}, U_{b}^{j}, U_{a}^{j^{\prime}}$, and $U_{b}^{j^{\prime}}$, based on the consumer utility function, can be expressed as follows:

$$
\begin{aligned}
& U_{a}^{j}=\left(-\alpha^{d_{p a}}+k_{j}\right) * p_{a}+\left(\beta^{\left|d_{q a}\right|}+\varepsilon_{j}\right) * q_{a}, \\
& U_{b}^{j}=\left(-\alpha^{d_{p b}}+k_{j}\right) * p_{b}+\left(\beta^{\left|d_{q b}\right|}+\varepsilon_{j}\right) * q_{b}, \\
& U_{a}^{j^{\prime}}=\left(-\alpha^{d_{p a}^{\prime}}+k_{j}\right) * p_{a}+\left(\beta^{\left|d_{q a}^{\prime}\right|}+\varepsilon_{j}\right) * q_{a}, \\
& U_{b}^{j^{\prime}}=\left(-\alpha^{d_{p b}^{\prime}}+k_{j}\right) * p_{b}+\left(\beta^{\left|d_{q b}^{\prime}\right|}+\varepsilon_{j}\right) * q_{b} .
\end{aligned}
$$

In the analysis above, if the decoy effect happens after the entry of decoy product $c$, it satisfies $U_{a}^{j^{\prime}}-U_{b}^{j}>0$ according to the given location of product $a\left(q_{a}, p_{a}\right)$, product $b\left(q_{b}, p_{b}\right)$, and $E_{1}\left(\bar{q}_{1}, \bar{p}_{1}\right)$. The location of $E_{2}\left(\bar{q}_{2}, \bar{p}_{2}\right)$ can also be calculated. In addition, $U_{b}^{j}-U_{a}^{j}=e$, where $e$ is a positive number that infinitely advances to zero, so what we need to do is to make sure $U_{a}^{j^{\prime}}-U_{a}^{j}-\left(U_{b}^{j^{\prime}}-U_{b}^{j}\right)>0$.

According to the location of product $a\left(q_{a}, p_{a}\right)$, product $b\left(q_{b}, p_{b}\right)$, and $E_{1}\left(\bar{q}_{1}, \bar{p}_{1}\right)$, the area for decoy products can be divided into 16 regions, as shown in Figure 1(a). When $E_{2} E_{2}\left(\bar{q}_{2}, \bar{p}_{2}\right)_{j^{\prime}}$ is located in these 16 regions, the corresponding $U_{a}^{j^{\prime}}-U_{a}^{j}-\left(U_{b}^{j^{\prime}}-U_{b}^{j}\right)$ can be calculated separately, expressed as the following formula:

$$
\begin{aligned}
& U_{a}^{j^{\prime}}-U_{a}^{j}-\left(U_{b}^{j^{\prime}}-U_{b}^{j}\right) \\
& =\left(\alpha^{d_{p a}}-\alpha^{d_{p a}^{\prime}}\right) * p_{a}-\left(\alpha^{d_{p b}}-\alpha^{d_{p b}^{\prime}}\right) * p_{b}+\left(\beta^{\left|d_{q a}^{\prime}\right|}-\beta^{\left|d_{q a}\right|}\right) * q_{a}-\left(\beta^{\left|d_{q b}^{\prime}\right|}-\beta^{\mid d_{q b}} \mid\right) * q_{b}>0 .
\end{aligned}
$$

By calculation, when $E_{2}\left(\bar{q}_{2}, \bar{p}_{2}\right)$ is located in region $S 11$, it satisfies $p_{a}>\bar{p}_{2}>\bar{p}_{1}>p_{b}$ and $q_{a}>\bar{q}_{2}>\bar{q}_{1}>q_{b}$, where $\left|d_{p a}\right|=\left|d_{p b}\right|,\left|d_{q a}\right|=\left|d_{q b}\right|, d_{p a}>d_{p a}^{\prime}, d_{q_{A}}>d_{q_{A}}^{\prime},\left|d_{p b}\right|<\left|d_{p b}^{\prime}\right|$, $\left|d_{q b}\right|<\left|d_{q b}^{\prime}\right|, \quad d_{p b}-d_{p b}^{\prime}=d_{p a}-d_{p a}^{\prime}=\bar{p}_{2}-\bar{p}_{1}>0, \quad$ and $d_{q b}-d_{q b}^{\prime}=d_{q a}-d_{q a}^{\prime}=\bar{q}_{2}-\bar{q}_{1}>0$.
It can be seen from the first chart in Figure 2 that $\left(\alpha^{d_{p a}^{\prime}}-\right.$ $\left.\alpha^{d_{p a}}\right) p_{a}>\left(\alpha^{d_{p b}^{\prime}}-\alpha^{d_{p b}}\right) p_{b} \quad$ can be obtained when $p_{a}>\bar{p}_{1}>\bar{p}_{2}>p_{b}, d_{p b}-d_{p b}^{\prime}=d_{p a}-d_{p a}^{\prime}, \quad \alpha^{d} \quad p^{\prime} a-\alpha^{d_{p a}}>$ $\alpha^{d_{p b}^{\prime}}-\alpha^{d_{p b}}>0$, and $\alpha^{d_{p a}^{\prime}}-\alpha^{d_{p a}}>\alpha^{d_{p b}^{\prime}}-\alpha^{d_{p b}}>0$, which means $\left(\alpha^{d_{p a}}-\alpha^{d_{p a}^{\prime}}\right) p_{a}-\left(\alpha^{d_{p b}}-\alpha^{d_{p b}^{\prime}}\right) p_{b}>0$. As shown in the second 
TABLE 1: The difference between the price/quality and the mean of the product category in scenarios with and without a decoy.

\begin{tabular}{lr}
\hline Equations & Equations \\
\hline$d_{p a}=p_{a}-\left(p_{a}+p_{b}\right) / 2=p_{a}-\bar{p}_{1}$ & $d_{q a}=q_{a}-\left(q_{a}+q_{b}\right) / 2=q_{a}-\bar{q}_{1}$ \\
$d_{p b}=p_{b}-\left(p_{a}+p_{b}\right) / 2=p_{b}-\bar{p}_{1}$ & $d_{q b}=q_{b}-\left(q_{a}+q_{b}\right) / 2=q_{b}-\bar{q}_{1}$ \\
$d_{p a}^{\prime}=p_{a}-\left(p_{a}+p_{b}+p_{c}\right) / 3=p_{a}-\bar{p}_{2}$ & $d_{q a}^{\prime}=q_{a}-\left(q_{a}+q_{b}+q_{c}\right) / 3=q_{a}-\bar{q}_{2}$ \\
$d_{p b}^{\prime}=p_{b}-\left(p_{a}+p_{b}+p_{c}\right) / 3=p_{b}-\bar{p}_{2}$ & $d_{q b}^{\prime}=q_{b}-\left(q_{a}+q_{b}+q_{c}\right) / 3=q_{b}-\bar{q}_{2}$ \\
\hline
\end{tabular}

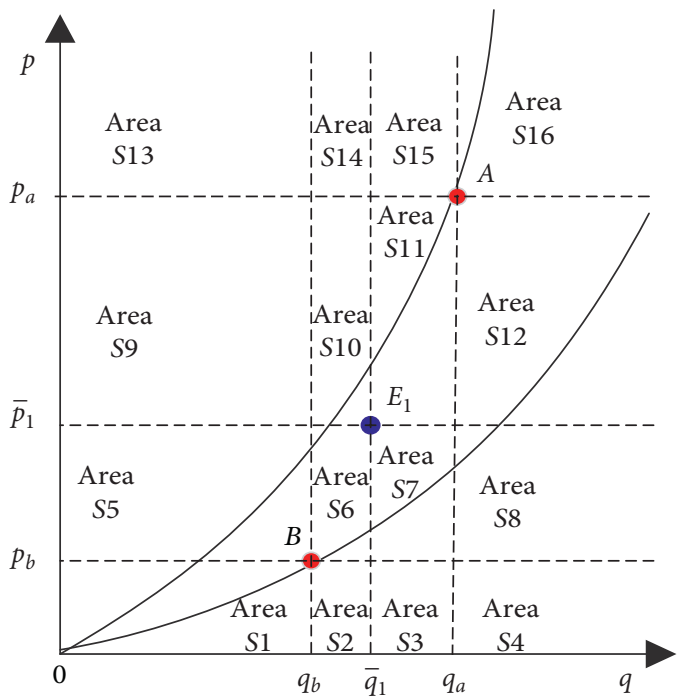

(a)

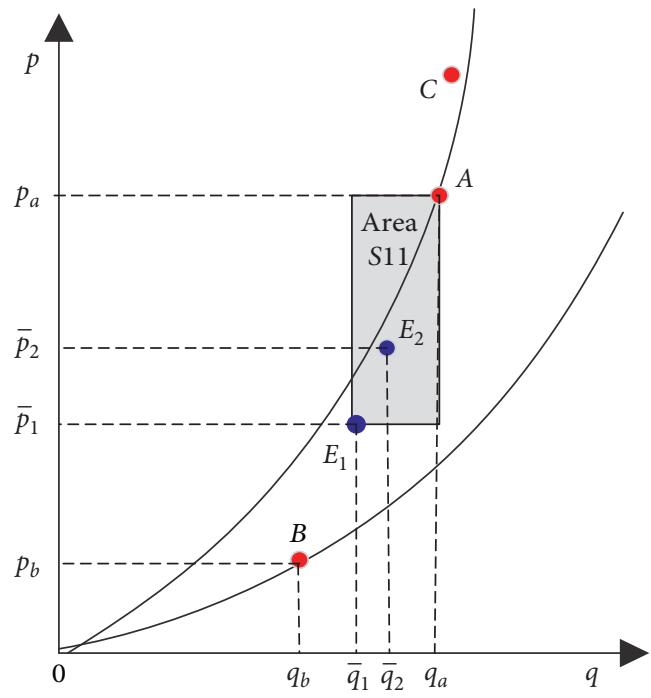

(b)

Figure 1: (a) Area divided. (b) Area of $E_{2}$.

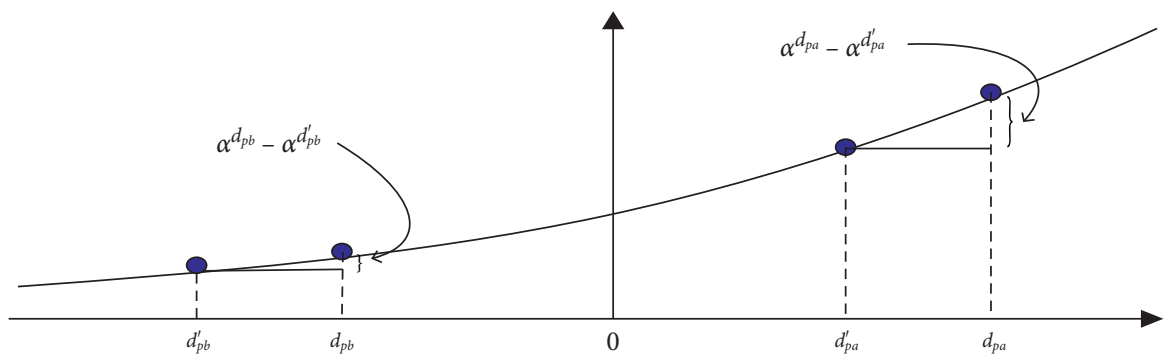

(a)

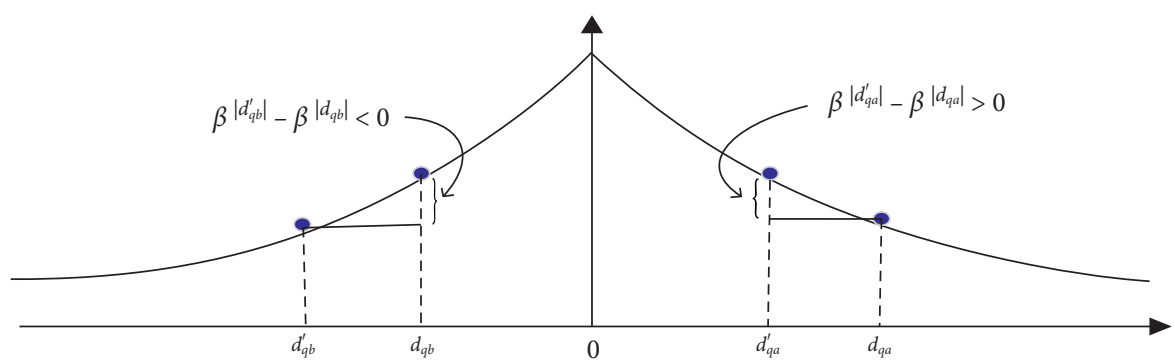

(b)

FIGURE 3: Charts of price sensitivity and quality sensitivity.

chart in Figure 2, if $0<\beta<1, \quad d_{q b}-d_{q b}^{\prime}=d_{q a}-d_{q a}^{\prime}$, $\beta^{\left|d_{q a}^{\prime}\right|}-\beta^{\left|d_{q a}\right|}>0, \quad \beta^{\left|d_{q b}^{\prime}\right|}-\beta^{\left|d_{q b}\right|}<0$, and $\quad q_{a}>q_{b}$, then $\left(\beta^{\left|d_{q a}^{\prime}\right|}-\beta^{\left|d_{q a}\right|}\right) * q_{a}-\left(\beta^{\left|d_{q b}^{\prime}\right|}-\beta^{\left|d_{q b}\right|}\right) * q_{b}>0 . \quad$ Therefore,
$U_{a}^{j^{\prime}}-U_{a}^{j}-\left(U_{b}^{j^{\prime}}-U_{b}^{j}\right)>0$ can be calculated from formula (11). Finally, after the entry of decoy product $c$, we can draw the conclusion that $U_{a}^{j^{\prime}}>U_{b}^{j^{\prime}}$ and $U_{b}^{j}>U_{a}^{j}$ when $E_{2}$ is located in 
region $S 11$, and consumer $j$ 's preference will shift from product $b$ to product $a$.

In addition, a consumer's preference also shifts from product $b$ to product $a$ when $E_{2}$ is located in region $S 15$. However, because region $S 15$ has no boundaries, we do not discuss it here. In other regions, either there is no change in consumer $j$ 's purchasing decisions or we are not sure whether they change, so we do not discuss these regions either.

When $E_{2}\left(\bar{q}_{2}, \bar{p}_{2}\right)$ is located in region $S 11$, the decoy effect of product $c$ will impact some consumers. In this case, the price interval and quality interval of decoy product $c$ can be calculated. The quality interval of $E_{2}$ is $q_{a}>\bar{q}_{2}>\bar{q}_{1}$; that is, $q_{a}>\left(q_{a}+q_{b}+q_{c}\right) / 3>\left(q_{a}+q_{b}\right) / 2$, which also means $2 q_{a}-q_{b}>q_{c}>\left(q_{a}+q_{b}\right) / 2$, so $q_{c}$ is $\left[\left(q_{a}+q_{b}\right) / 2,2 q_{a}-q_{b}\right]$. The price interval of $E_{2}$ is $p_{a}>\bar{p}_{2}>\bar{p}_{1}$; that is, $p_{a}>\left(p_{a}+p_{b}+p_{c}\right) / 3>\left(p_{a}+p_{b}\right) / 2$, which also means $2 p_{a}-p_{b}>p_{c}>\left(p_{a}+p_{b}\right) / 2$, so $p_{c}$ is $\left[\left(p_{a}+p_{b}\right) / 2,2 p_{a}-p_{b}\right]$. The range of $q_{c}$ and $p_{c}$ is shown in Figure 3.

\section{Initial Parameters and Experimental Scenario Setting}

Under the background of true decoy strategies, this paper studies the difference in the decoy effects generated by different combinations of decoy price and quality. Also, the evolution of target product sales is discussed. On this basis, the results of the same true decoy strategy as affected by the characteristics of the consumer network can be analyzed. Consumer neighbor nodes, reconnection probabilities, and herd strengths of consumer social networks are the most important parameters used to describe consumer interaction networks, which have very profound management significance. Three experiments were set up to explore the results of different true decoy strategies in scenarios with different values for nodes $K$, reconnection probability $P_{r}$, and herd mentality $\theta_{j}$, respectively.

The basic parameters of the experimental model are kept constant. In the first experiment, the basic parameters are $K=2,4$, and 8 . In the second experiment, the basic parameters are $P_{r}=0.1,0.3$, and 0.5 . In the third experiment, the basic parameters are $\theta_{j} \in(0,0.1), \theta_{j} \in(0.2,0.3)$, and $\theta_{j} \in(0.4,0.5)$. Regarding the value of these three parameters, the value of the model parameter constructed by the multiagent modeling method should be within a certain reasonable range. The consumer interactive network in this article has the characteristics of a small-world network. For example, the number of consumer theoretical nodes should be an even number. For the setting of its value, refer to Bhole and Hanna [21]. We have discussed the evolution of the decoy effect when the number of neighbor nodes is 2,4 , and 8 . The value of the reconnection probability is between 0 and 1. Specific settings refer to Bohlmann et al. [22]. $p_{r}=0.1$ indicates that other consumers have less influence on the consumer and $p_{r}=0.5$ indicates that other consumers have a higher influence on the consumer. Therefore, this paper considers three cases of reconnection probability of $0.1,0.3$, and 0.5 . The value of herd strengths of consumer social networks is based on Lee et al. [23]. In the simulation model proposed by Lee et al., consumers had a herd mentality in the evaluation of new products, and the evaluation of new products is generated through interaction. Therefore, this article divides the value of $\theta_{j}$ into three intervals to show the heterogeneity of consumers' influence by others. It is assumed that the price interval of the decoy product is [49, 141] and the quality interval of the decoy product is [52, 150]. In this case, there are 21 different prices and 21 levels of quality. The gap between the price is 4.4 , and the gap between the quality is 4.67. Taking into account the possible combinations of price and quality for the decoy product, there are 441 kinds of true decoy strategies. The experiment is run separately for each of these true decoy strategies, given $K, P_{r}$, and $\theta_{j}$. To eliminate randomness in the process of experiments and improve the stability and validity of the experimental results, we run each experiment 30 times. Finally, we carried out a statistical analysis of the experimental results obtained; the initial values of the model parameters are shown in Table 2.

\section{Simulation Results}

5.1. The Influence of Consumer Neighbor Nodes. The sales of the target product (product $a$ ) are used to characterize the effectiveness of the true decoy strategy. Figure 4 shows the evolution of results of the true decoy strategy at different time periods $(T=1, T=5$, and $T=10)$ in the scenarios with different numbers of nodes $(K=2, K=4$, and $K=8)$. As shown in Figure 4, corresponding to the different true decoy strategies, the results show a certain degree of difference, hierarchy, nonlinearity, and regularity. The three figures in the first row of Figure 4 represent the sales of product $a$ in the initial state $(T=1)$ when the neighbor node is $K=2, K=4$, and $K=8$. As can be seen from Figure 4, centered on the price and quality of product $a$, when the price of decoy product $c$ is equal to the price of product $a$ and kept constant, the sales of product $a$ rise as the quality of decoy product $c$ declines, and the sales fall as the quality of decoy product $c$ improves. When the quality of decoy product $c$ is equal to the quality of product $a$ and kept constant, the sales of product $a$ rise as the price of decoy product $c$ declines, and the sales fall as the price of decoy product $c$ rises. Based on this, four regions, $A, B, C$, and $D$, for true decoy strategies are formed.

Based on the price and quality of target product $a$, the decoy area can be classified into four categories: Class A (the price and quality of decoy product $c$ are both lower than those of the target), Class B (the price of decoy product $c$ is lower than that of the target while the quality of the decoy is higher than that of the target), Class $C$ (the price and quality are both higher than those of the target), and Class D (the price of the decoy is higher than that of the target while the quality of the decoy is lower than that of the target). These are shown in Figure 4.

According to Figure 4 , in the initial state $(T=1)$, the effect of the true decoy strategy is the same in the scenarios with neighbor nodes $(K=2, K=4$, and $K=8)$ when there is no interaction between consumers; when there are interactions between consumers and the period is the same, the effectiveness of the true decoy strategy varies with the 


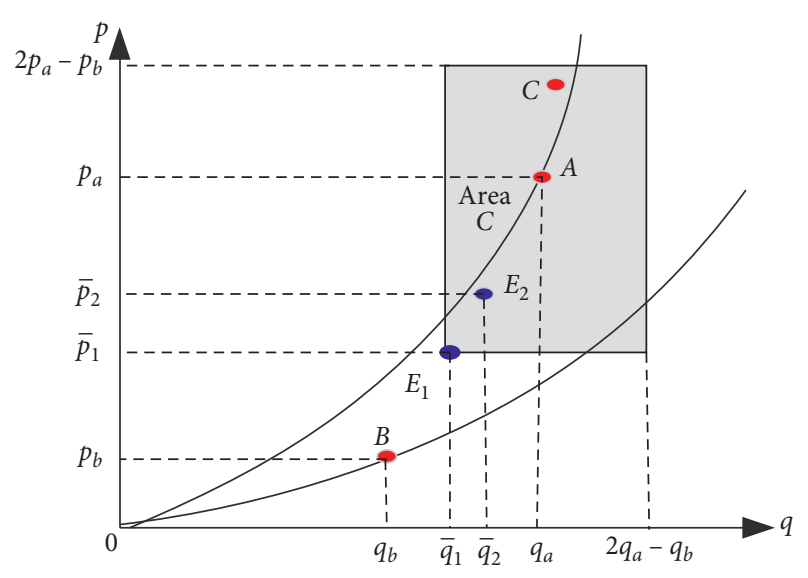

Figure 3: Interval of decoy product $c$.

number of neighbor nodes. For example, at $T=10$, the effectiveness of a Class $\mathrm{C}$ true decoy strategy is strong when the number of neighbor nodes is $K=2$, and it improves when $K=8$; when the number of neighbor nodes is the same, the effectiveness of different true decoy strategies shows different evolution trends over time. In the $K=2$ scenario, the effectiveness of a Class A true decoy strategy is similar to the effectiveness of a Class $C$ true decoy strategy at time $T=1$. However, with the change of time, the effect of the Class $\mathrm{C}$ true decoy strategy improves while the effectiveness of the Class A true decoy strategy weakens at time $T=10$.

To explore the evolution of the effectiveness trend for true decoy strategies with different numbers of neighbor nodes, some points have been selected in the decoy areas. For research convenience, we set the true decoy strategy (Q: 72, $P: 67)$ in the region of Class $A,(Q: 91.5, P: 67)$ in the region of Class $B,(Q: 91.5, P: 95)$ in the region of Class $\mathrm{C}$, and $(Q: 72, P: 95)$ in the region of Class D.

Based on the concept that there are A, B, C, and D kinds of true decoy strategies, Figure 5 shows the evolution trend for sales of target product $a$, decoy product $c$, and competing product $b$ when the number of neighbor nodes is $K=2$, $K=4$, and $K=8$. According to Figure 5 , in the scenarios with different nodes, the sales of target product $a$, decoy product $c$, and competing product $b$ show similar evolution trends under the same true decoy strategy. In the initial state $(T=1)$, the sales of target product $a$ under the A and B true decoy strategies are higher than those under the $\mathrm{C}$ and $\mathrm{D}$ true decoy strategies. However, as the period changes, the sales of product $a$ under true decoy strategies in Classes A and $\mathrm{B}$ decline while they rise under true decoy strategies in Classes C and D. Through the analysis, we found that the reason for this phenomenon is the existence of decoy product $c$. Under Classes A and B true decoy strategies, the sales of decoy product $c$ increase as the period changes. Decoy product $c$ does not play a good role, and it gradually seizes market share, reducing the sales of product $a$. However, Classes $\mathrm{C}$ and $\mathrm{D}$ true decoy strategies play well. In addition, no matter which kind of true decoy strategy, the sales of product $b$ decrease.
In the scenarios for the four kinds of true decoy strategies, Class A, Class B, Class C, and Class D, Figure 6 shows the sales and evolution trends of target $a$, decoy $c$, and competing product $b$ when the number of nodes is $K=2$, $K=4$, and $K=8$. As shown in Figure 6 , in the scenarios with different true decoy strategies, the evolution trends for sales of the three products, $a, b$, and $c$, show a certain regularity with the change in neighbor nodes. When the sales volume of a product is on the rise, the speed of sales increases as the number of neighbor nodes rises. When the sales volume of a product shows a downward trend, the speed of sales also increases with the number of neighbor nodes. Based on this, we can conclude that an increase in neighbor nodes will aggravate the evolutionary trend of product sales.

According to the experimental results, first of all, the setting of the true decoy strategy has an important impact on consumer purchasing decisions, and an unreasonable setting may even have a negative result. For example, true decoy strategies in Classes $C$ and D play a good role, and the sales of target product $a$ increase. However, true decoy strategies in Classes A and B do not have positive results. Decoy product $c$ seizes the market and causes the sales of product $a$ to decline. Secondly, for the same true decoy strategy, an increase in the number of neighbor nodes will aggravate the evolution of product sales through the process of consumer interaction. Therefore, in the process of product marketing, companies should clearly define their purpose and set suitable true strategies. At the same time, taking the interaction between consumers into account, companies can take certain measures to strengthen the effectiveness of the decoy strategy by raising awareness among other consumers about the experience and feelings evoked from product use.

5.2. The Influence of Reconnection Probabilities. Figure 7 shows the evolution trend for the effectiveness of true decoy strategies at different times $(T=1, T=5$, and $T=10)$ in scenarios with different reconnection probabilities $\left(P_{r}=0.1\right.$, $P_{r}=0.3$, and $\left.P_{r}=0.5\right)$. It can be seen from Figure 7 that, corresponding to the different values of the price and quality of the true decoy, the sales volume of target product $a$ and its evolution trend show a certain difference, level, nonlinearity, and regularity. When $T=1$, the sales of product $a$ under the same true decoy strategy are the same in scenarios with different reconnection probabilities $\left(P_{r}=0.1, P_{r}=0.3\right.$, and $\left.P_{r}=0.5\right)$. With time changes, the effectiveness of the same strategy shows different evolution trends under different reconnection probabilities.

As shown in Figure 7, considering the interaction between consumers, when the period is the same, the effectiveness of the same true decoy strategy presents different evolution trends with different reconnection probabilities. For example, when $T=10$, the effectiveness of the Class $C$ true decoy strategy is ordinary in the scenario with reconnection probability $P_{r}=0.1$ and improves when $P_{r}=0.5$. When the reconnection probability is the same, the effectiveness of different true decoy strategies shows different evolution trends with time changes. For example, when $P_{r}=0.3$, with time changes, the sales of target product $a$ in 
TABLE 2: Initial values of the basic parameters of the model.

\begin{tabular}{lccc}
\hline Parameter & Range of value & Distribution & References/description \\
\hline$q_{a}$ & 85 & Constant & {$[1,24]$} \\
$p_{a}$ & 72 & Constant & {$[1,24]$} \\
$q_{b}$ & 20 & Constant & {$[3]$} \\
$p_{b}$ & 17.6 & Constant & {$[3]$} \\
$q_{c}$ & {$[52,150]$} & Constant & {$[16,24]$} \\
$p_{c}$ & {$[49,141]$} & Constant & {$[16,24]$} \\
$\partial_{j}$ & $N(3.6,1.6)$ & Normal distribution & {$[16]$} \\
$\beta_{j}$ & $N(0.6,0.2)$ & Normal distribution & {$[16]$} \\
$\theta_{j}$ & $U[0,0.5]$ & Uniform distribution & {$[23]$} \\
$p_{r}$ & 0.3 & Constant & {$[22]$} \\
$\mathrm{SE}_{j}$ & $N(500,75)$ & Normal distribution & {$[23]$} \\
$K$ & 4 & Constant & {$[21]$} \\
$N$ & 5000 & Constant & {$[16]$} \\
\hline
\end{tabular}
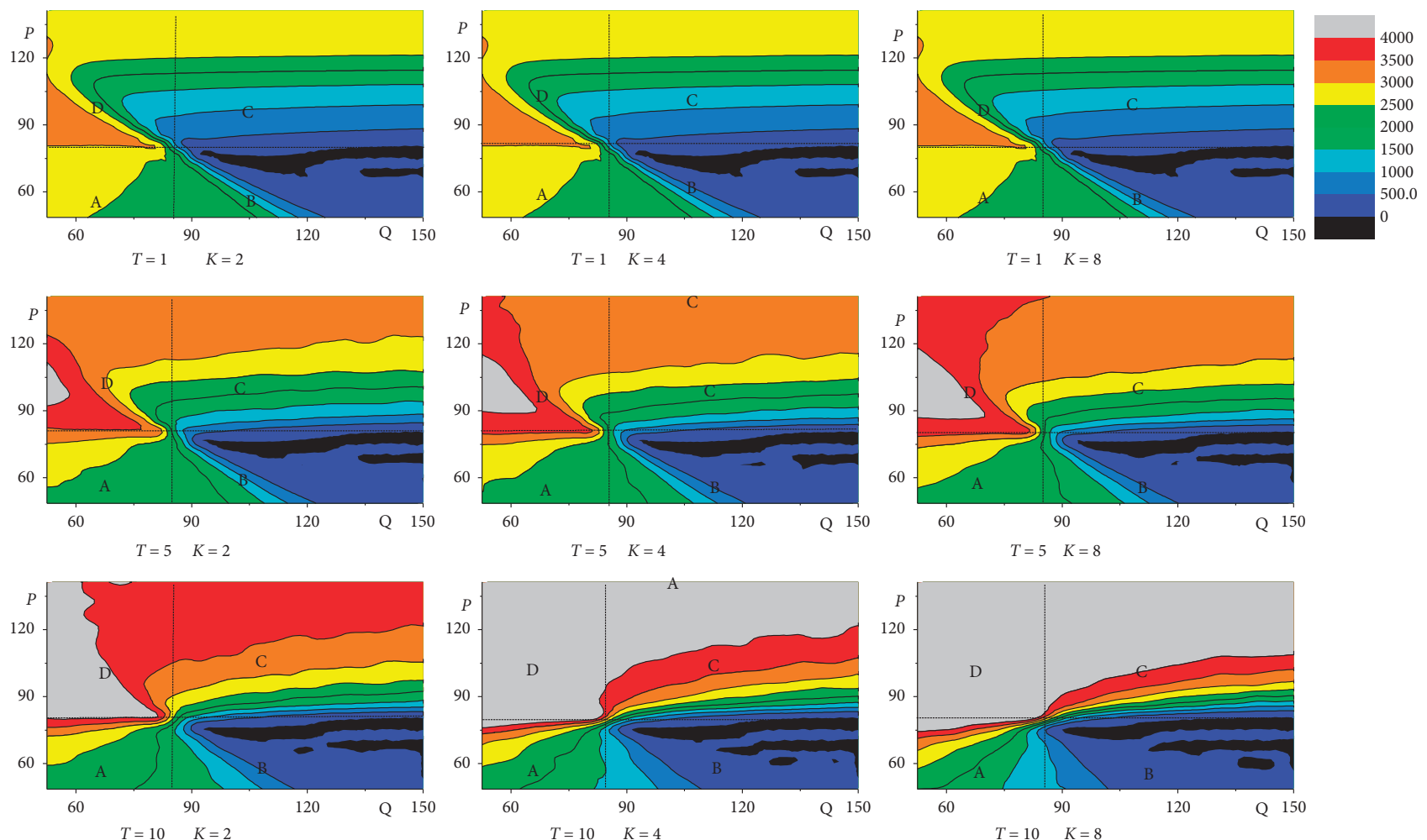

FIGURE 4: Effectiveness of true decoy strategies in scenarios with different neighbor node values.

the Class A true decoy strategy decline, and they remain at a low level in the Class B strategy, while, in Classes C and D true decoy strategies, the sales of target product $a$ increase with time changes.

To explore the evolution trend for the sales and the effects of reconnection probabilities on the true decoy strategies, some points have been selected in the decoy areas. Based on the concepts of A, B, C, and D kinds of true decoy strategies, Figure 8 shows the evolution trend for sales of target product $a$, decoy product $c$, and competing product $b$ when the reconnection probability is $P_{r}=0.1, P_{r}=0.3$, and $P_{r}=0.5$. As can be seen in Figure 8 , in the scenarios with different reconnection probabilities, the sales of target product $a$, decoy product $c$, and competed product $b$ show a similar evolution trend under the same true decoy strategy. In Classes A and B true decoy strategies, the sales of product $a$ gradually decrease as time changes, while the sales of decoy $c$ increase as time changes. In this situation, we can find that the true decoy strategies of Classes A and B do not play a positive role, even causing decoy $c$ to take the place of target product $a$. On the contrary, the true decoy strategies of Classes $C$ and D play a positive role, and with time changes, the sales of target product $a$ gradually increase.

Figure 9 shows the evolution trend for sales of target product $a$, decoy product $c$, and competing product $b$, when the reconnection probability is $P_{r}=0.1, P_{r}=0.3$, and $P_{r}=0.5$, for A, B, C, and D kinds of true decoy strategies. As shown in Figure 9, the sales of target product $a$, decoy 

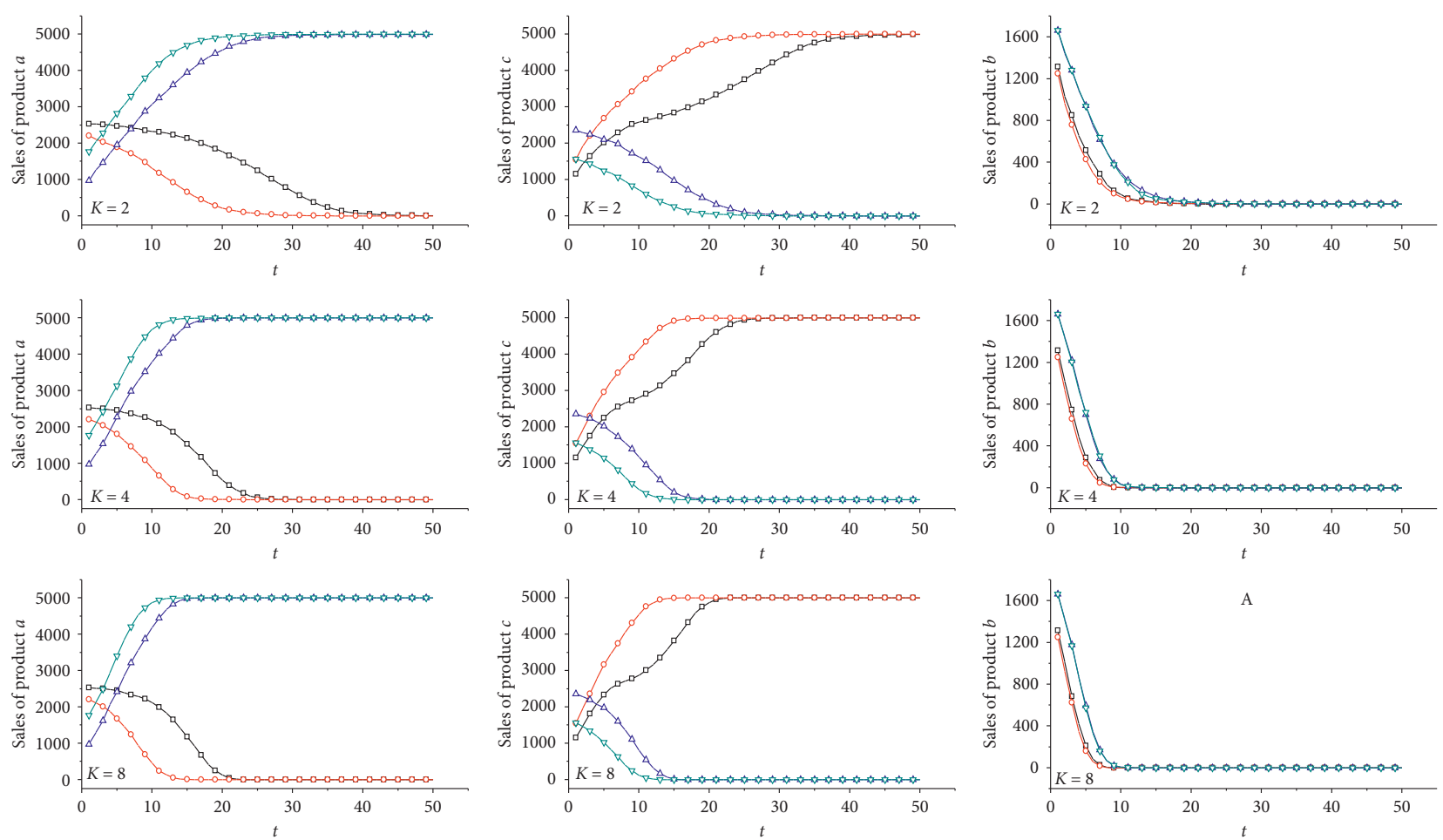

$\neg-$ Decoy strategy of class A $(Q ; 72, P ; 67)$

$-\infty$ Decoy strategy of class B $(Q: 91.5, P: 67)$

$\triangle-$ Decoy strategy of class $\mathrm{C}(Q ; 91.5, P: 95)$

$\rightarrow-$ Decoy strategy of class $\mathrm{D}(Q: 72, P: 95)$

Figure 5: Evolution trends for sales of products $a, c$, and $b$ under different true decoy strategies in scenarios with different neighbor node values.
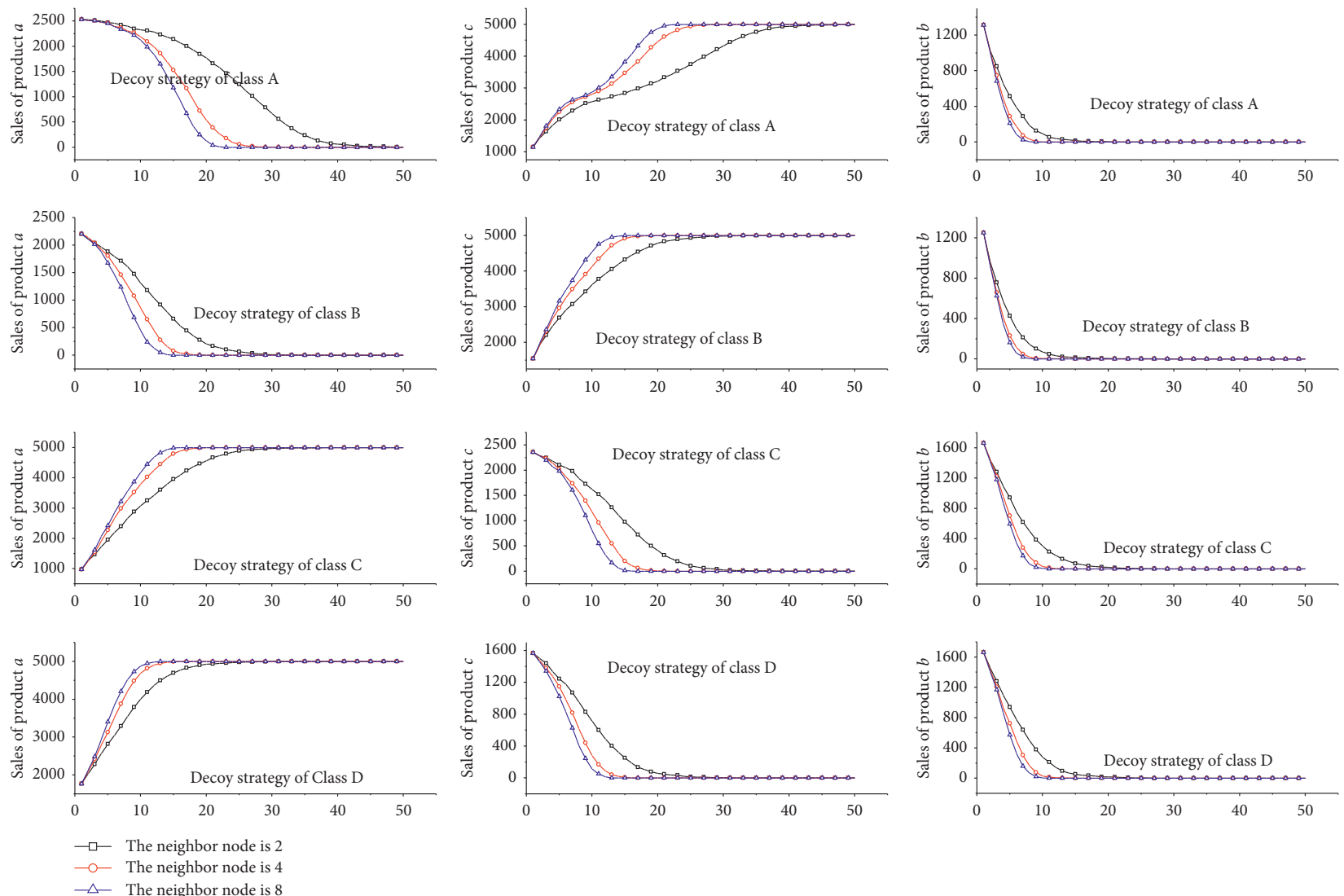

Figure 6: Evolution trends for sales of products $a, c$, and $b$ under the four kinds of true decoy strategies in scenarios with different neighbor node values. 

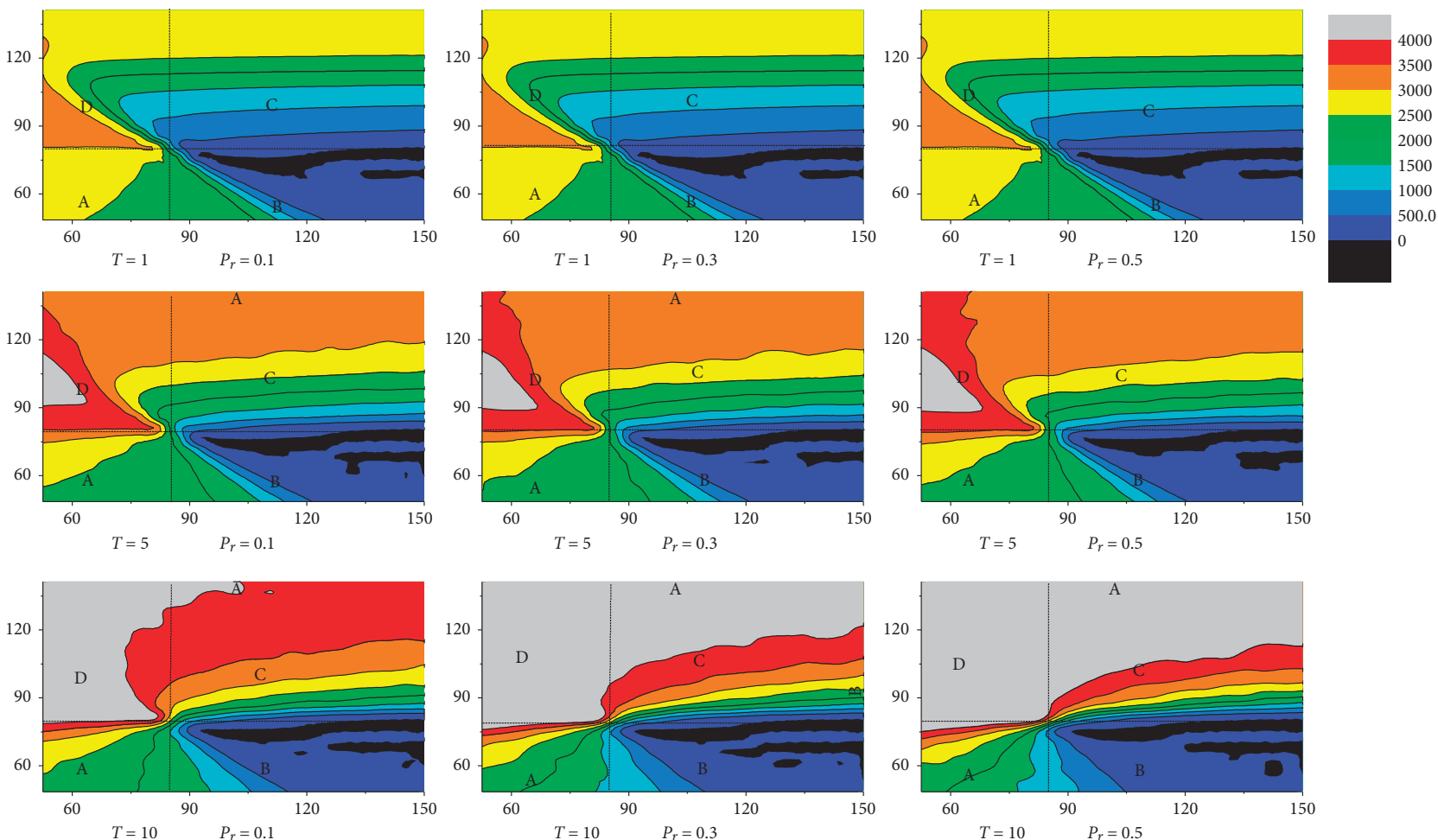

FIGURE 7: Effectiveness of true decoy strategies in scenarios with different reconnection probabilities.
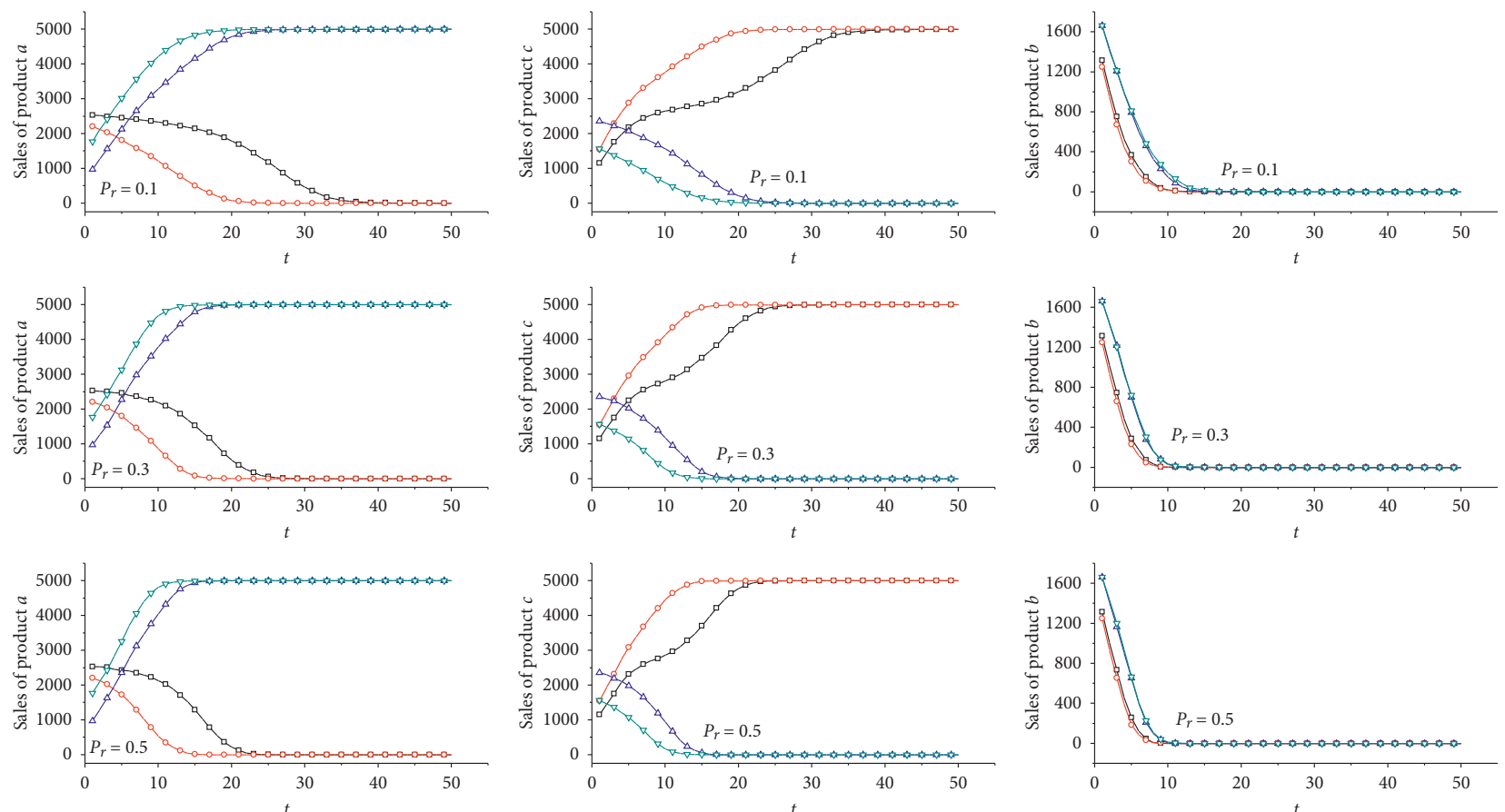

$$
\begin{aligned}
& -\square \text { Decoy strategy of class A }(Q ; 72, P ; 67) \\
& -\triangle \text { Decoy strategy of class C }(Q ; 91.5, P: 95) \\
& -\square \text { Decoy strategy of class B }(Q: 91.5, P: 67) \\
& \neg-\text { Decoy strategy of class D }(Q: 72, P: 95)
\end{aligned}
$$

FigURE 8: Evolution trends for sales of products $a, c$, and $b$ under different true decoy strategies in scenarios with different reconnection probabilities. 

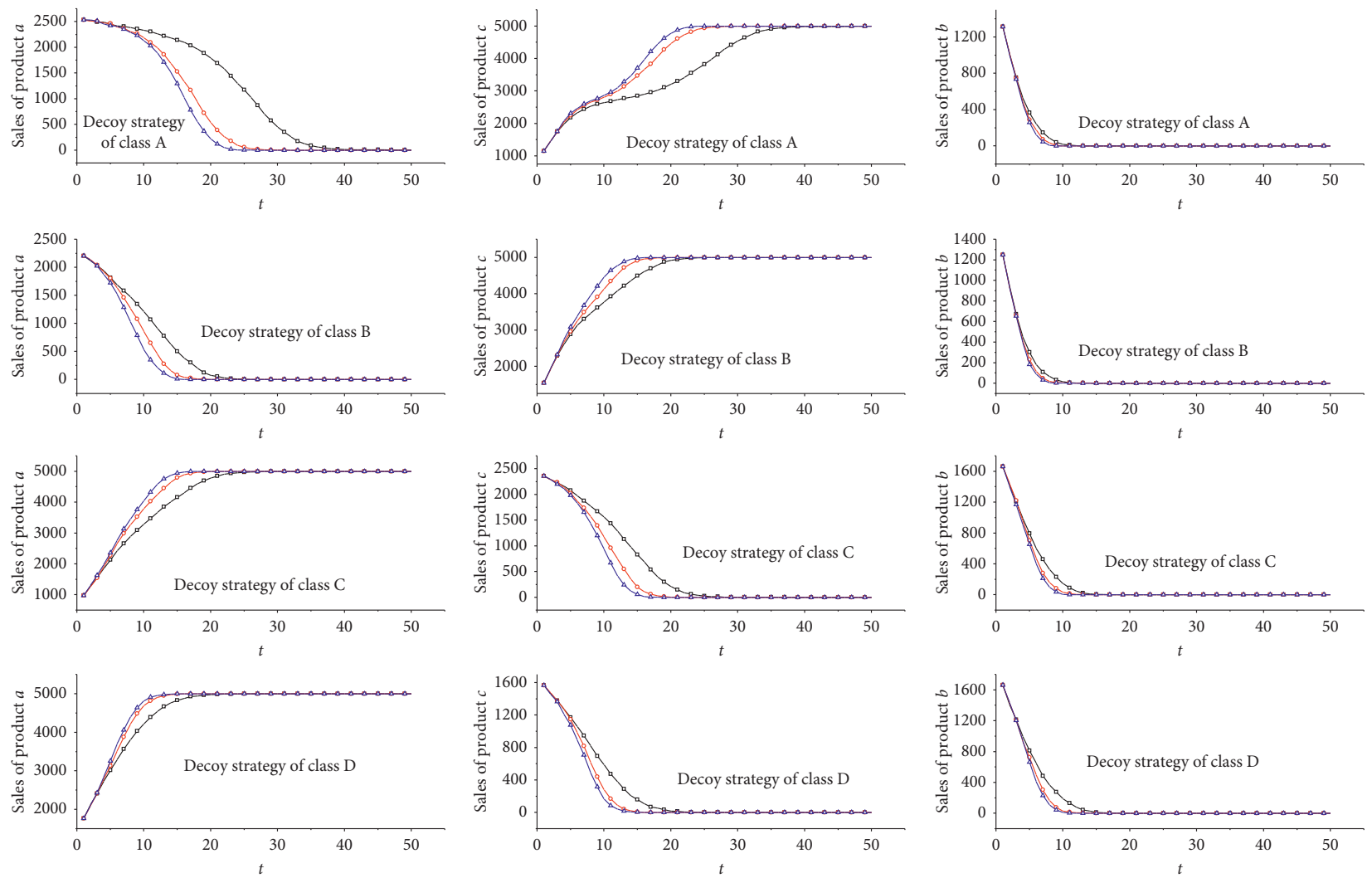

$\square-$ The reconnection probability is 0.1
$-\square$ The reconncetion probability is 0.3
$\triangle-$ The reconnection probability is 0.5

Figure 9: Evolution trend for sales of products $a, c$, and $b$ under the four kinds of true decoy strategies in scenarios with different reconnection probabilities.

product $c$, and competing product $b$ show some regularity with changes in reconnection probabilities in scenarios with different true decoy strategies. In the scenario where the sales of products are on the rise, the rate of the increase in sales increases as the reconnection probability increases. Also, when product sales show a downward trend, the rate of decline in sales increases with the reconnection probability. Based on this, we can conclude that an increase in reconnection probability will aggravate the evolution trend for product sales.

By comparing the experimental results, some conclusions in experiment one have been verified. First, the setting of true decoy strategies has an important impact on consumer purchasing decisions. An inappropriate true decoy strategy can not only play a negative role but also inhibit the sales of target products. Second, for the same true decoy strategy, an increase in the reconnection probability in the process of consumer interaction will aggravate the evolution of product sales. Therefore, companies should take measures to prevent consumers from interacting with fixed objects (e.g., family members and friends) in the purchase decision process and encourage them to seek new objects with which to understand and communicate, such as using information technology to show consumers evaluations by other consumers and to increase word of mouth.
5.3. The Influence of Herd Strengths. Figure 10 shows the evolution trend for the sales of target product $a$ at different times $(T=1, T=5$, and $T=10)$ in scenarios with different herd strengths $(\theta \in(0,0.1), \theta \in(0.2,0.3)$, and $\theta \in(0.4$, $0.5)$ ). As shown in Figure 10, corresponding to the settings of the different true decoy strategies, the sales volume of target product $a$ and its evolution trend show a certain difference, level, nonlinearity, and regularity. In the initial state $(T=1)$, the sales of product $a$ under the same true decoy strategy are the same in scenarios with different herd strengths. That means when there are no interactions between consumers at the beginning, consumers' herd behaviors have no impact on the effectiveness of the true decoy strategy.

However, the situation changes after considering interactions between consumers. The effectiveness of the same true decoy strategy varies with herd strength when the time is the same. For instance, at time $T=5$, the effectiveness of the true decoy strategy in Class $\mathrm{D}$ is not very good in the scenario with a lower herd strength $(\theta \in(0,0.1))$ while, in the scenario with a higher herd strength $(\theta \in(0.4,0.5))$, the effectiveness of the true decoy strategy in Class $\mathrm{D}$ is better. In addition, in scenarios with the same herd strength, the effectiveness (the sales of target product $a$ ) of the different true decoy strategies shows different evolution trends with time 

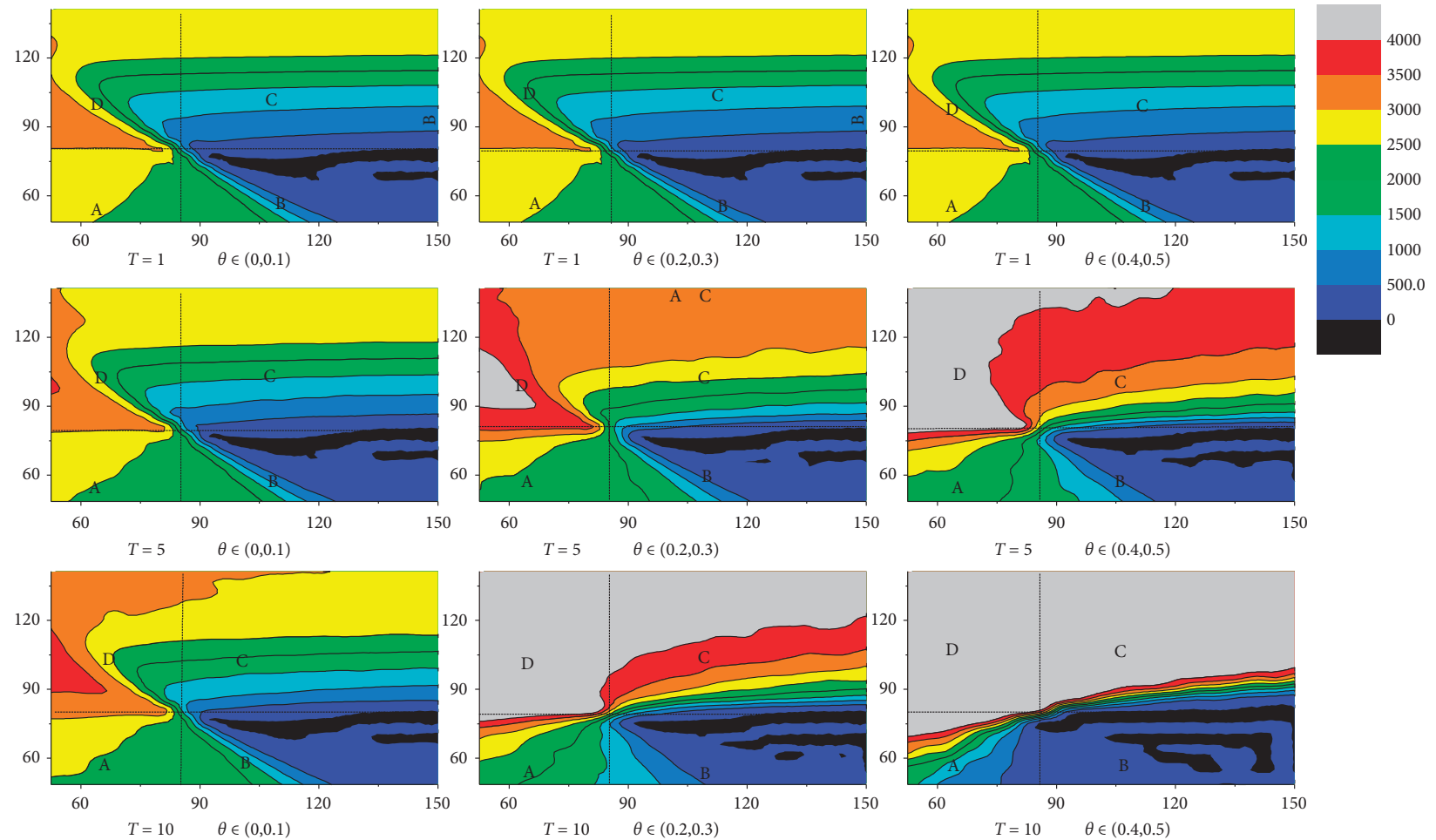

FiguRE 10: Effectiveness of true decoy strategies in scenarios with different herd strengths.

changes. For example, in the scenario with the herd strength $\theta \in(0.2,0.3)$ at time $T=5$, the effectiveness of the true decoy strategy in Class A worsens while the effectiveness of the true decoy strategy in Class D improves.

To explore the evolution trend for the sales in the scenarios with different herd strengths, some points have been selected in the A, B, C, and D decoy areas. Figure 11 shows the evolution trend for the sales of target product $a$, decoy product $c$, and competing product $b$ when the herd strength is $\theta \in(0,0.1), \theta \in(0.2,0.3)$, and $\theta \in(0.4,0.5)$. As shown in Figure 11, in the scenarios with different herd strengths, the sales of target product $a$, decoy product $c$, and competing product $b$ show a similar evolution trend under the same true decoy strategy. The sales of product $a$ in the true decoy strategies of Classes A and B gradually decrease as time changes while the sales in the true decoy strategies of Classes $\mathrm{C}$ and $\mathrm{D}$ increase as time changes. On the contrary, the sales of decoy $c$ in the true decoy strategies of Classes A and B gradually increase as time changes while the sales in the true decoy strategies of Classes $C$ and $D$ decrease as time changes. In addition, the sales of product $b$ decrease in all four kinds of true decoy strategies. In addition, we find that when the herd strength is small $(\theta \in(0,0.1))$, the evolution trend for sales of product $a$, product $b$, and product $c$ changes slowly while it changes quickly when the herd strength is big $(\theta \in(0.2,0.3), \theta \in(0.4,0.5))$.

Figure 12 shows the evolution trend for the sales of target product $a$, decoy product $c$, and competing product $b$ when the herd strength is $\theta \in(0,0.1), \theta \in(0.2,0.3)$, and $\theta \in(0.4,0.5)$. According to Figure 12, the sales of target product $a$, product $c$, and product $b$ show some regularity with changes in herd strength in the scenarios with different true decoy strategies. In the scenarios where the sales of products are on the rise, the rate of increase in sales increases as the herd strength increases. Also, in the scenarios where the sales of products show a downward trend, the rate of decline in sales increases as herd strength increases. Based on this, we can conclude that an increase in reconnection probability will aggravate the evolution trend for products sales, which also verifies some conclusions based on Figure 11.

By analyzing the results of the experiment, it can be concluded that consumer purchasing decisions are affected by decoys and interactions with other consumers. In a certain scenario, different true decoy strategies have different impacts on the sales of the target product (product $a$ ). In terms of effectiveness, however, for the same decoy strategy, an increase in herd strength will aggravate the evolution trend and form a path of dependence. Therefore, to ensure the sales and competitiveness of a target product, companies should set an appropriate true decoy strategy based on an understanding of herd effects.

\section{Conclusions}

The competition and diffusion of products in the market are of great significance to enterprises. In view of the various true decoy strategies constructed with combinations of price and quality and considering the interactions between consumers, this paper studies the evolution trend and differences in the effectiveness of different true decoy strategies. Based on the consumer network, the influence of neighbor 

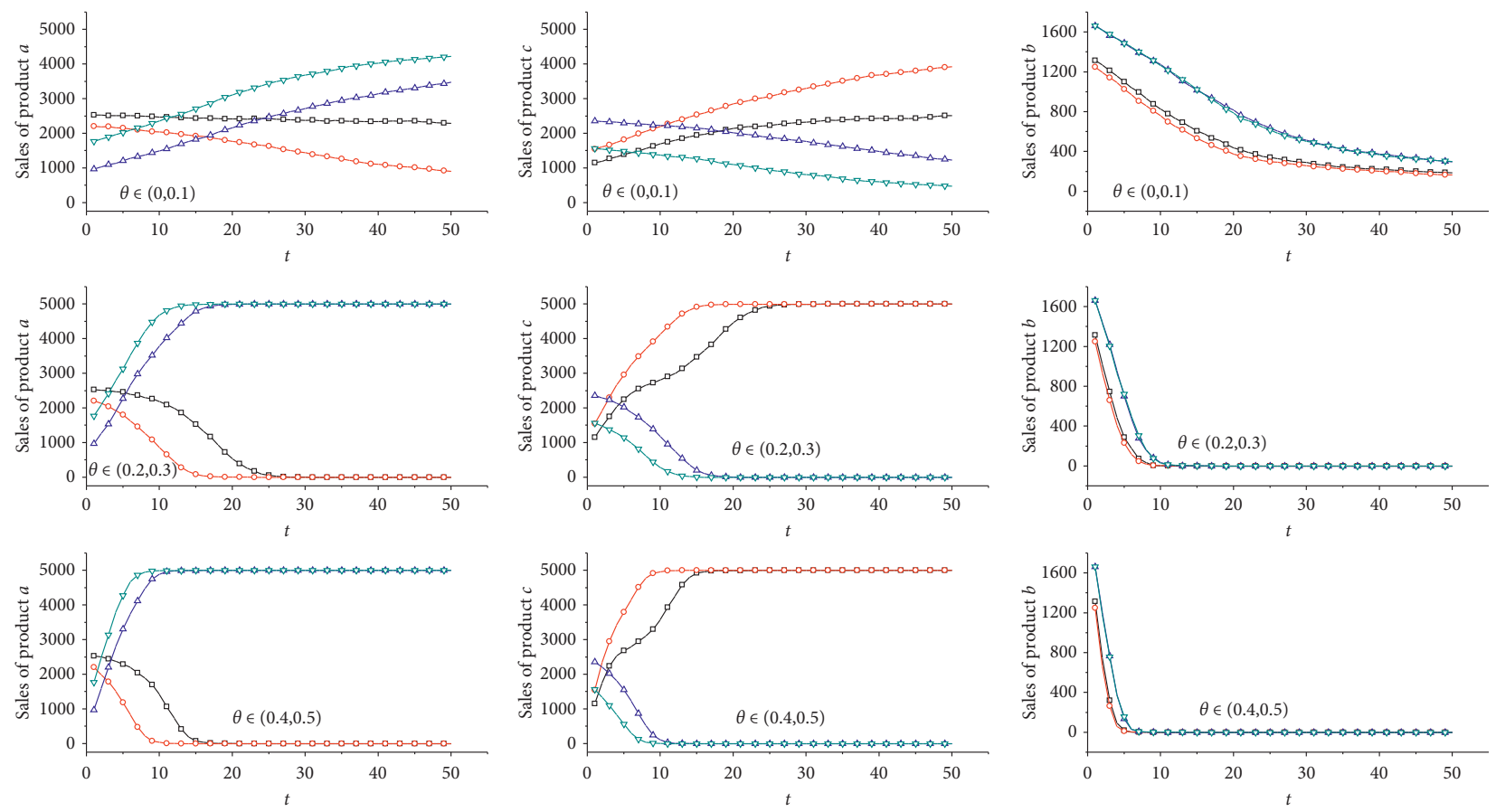

$\neg-$ Decoy strategy of class A $(Q ; 72, P ; 67)$ -O- Decoy strategy of class B $(Q: 91.5, P: 67)$ $\triangle$ Decoy strategy of class C (Q; 91.5, P. 95)

$\rightarrow-$ Decoy strategy of class D (Q: 72, $P: 95)$

FigURE 11: Evolution trends for sales of products $a, c$, and $b$ under different true decoy strategies in scenarios with different herd strengths.
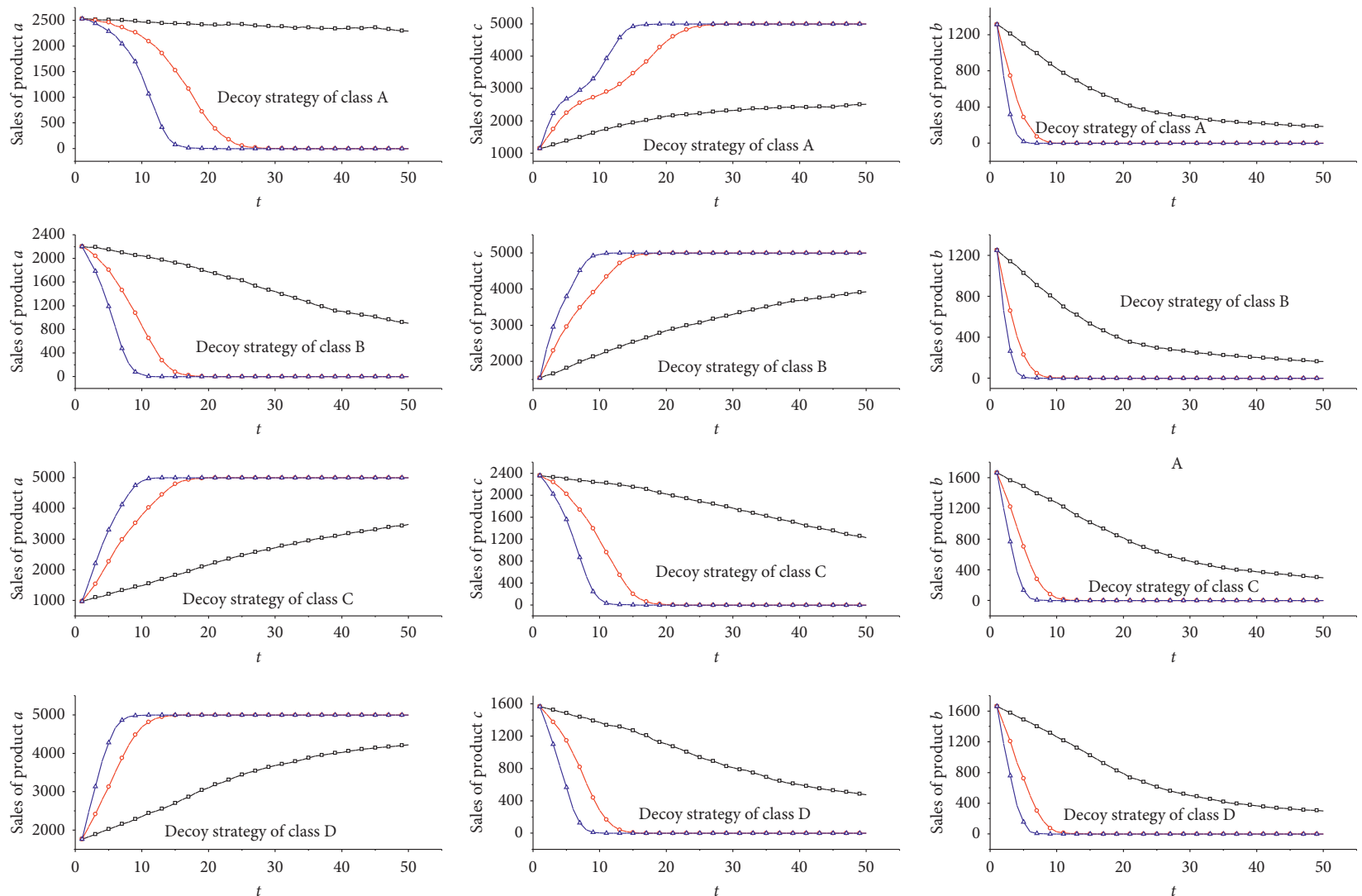

$\square$ The herd strength is $0-0.1$
$\square-$ The herd strength is $0.2-0.3$
$\square \quad$ The herd strength is $0.4-0.5$

FIGURE 12: Evolution trend for sales of products $a, c$, and $b$ under the four kinds of true decoy strategies in scenarios with different herd strengths. 
nodes, reconnection probabilities, and herd strengths on the effectiveness of true decoy strategies are explored through specific scenarios. The research conclusion is a description of the evolution trend of the decoy effect under different management scenarios, and then the corresponding management conclusions and enlightenments are obtained. It does not pay attention to the absolute value of the experimental results but to the analysis of the evolutionary trend. These will provide a theoretical basis for companies to take measures to improve the competitiveness of their target products.

The results of the experiment show that the competitiveness of a target product can be enhanced by setting an appropriate true decoy strategy. Otherwise, the decoy product cannot enhance the competitiveness of the target product; it may even take the place of the target product. In terms of the effectiveness of the same true decoy strategy, an increase in neighbor nodes, reconnection probabilities, and herd strengths in consumer networks can speed up the evolution trend of products. However, it does not change the original direction of the evolution trend. Therefore, when the market is favorable to a target product, companies should take measures to increase interaction between consumers and educate them about the experience and feelings of other consumers. To prevent consumers from interacting with fixed objects during the process of purchase decision-making, consumers are encouraged to seek new information and communicate with new people. In addition, companies should pay attention to the consumer herd effect and adjust the price and quality of their products according to the different trends and stages of effectiveness for the true decoy strategy.

\section{Data Availability}

The data used to support the findings of this study are available from the corresponding author upon request.

\section{Conflicts of Interest}

The authors declare that there are no conflicts of interest regarding the publication of this paper.

\section{Acknowledgments}

This work was supported by the National Natural Science Foundation of China (nos. 71971100, 71671078, 71501084, 71974081, and 71771127); Social Science Fund of Jiangsu Province (19GLB005 and 19GLB018); and the Key Project of Philosophy and Social Science Research in Colleges and Universities in Jiangsu Province (2018SJZDI052), sponsored by Qing Lan Project of Jiangsu Province and Youth Backbone Teacher Training Project of Jiangsu University.

\section{References}

[1] J. Huber, J. W. Payne, and C. Puto, "Adding asymmetrically dominated alternatives: violations of regularity and the similarity hypothesis," Journal of Consumer Research, vol. 9, no. 1, pp. 90-98, 1982.
[2] Y. Pan and D. R. Lehmann, "The influence of new brand entry on subjective brand judgments," Journal of Consumer Research, vol. 20, no. 1, pp. 76-86, 1993.

[3] T. B. Heath and S. Chatterjee, "Asymmetric decoy effects on lower-quality versus higher-quality brands: meta-analytic and experimental evidence," Journal of Consumer Research, vol. 22, no. 3, pp. 268-284, 1995.

[4] A. Dan and T. S. Wallsten, "Seeking subjective dominance in multidimensional space: an explanation of the asymmetric dominance effect," Organizational Behavior \& Human Decision Processes, vol. 63, no. 3, pp. 223-232, 1995.

[5] J. C. Pettibone and D. H. Wedell, "Examining models of nondominated decoy effects across judgment and choice," Organizational Behavior and Human Decision Processes, vol. 81, no. 2, pp. 300-328, 2000.

[6] I. Simonson, "Choice based on reasons: the case of attraction and compromise effects," Journal of Consumer Research, vol. 16, no. 2, pp. 158-174, 1989.

[7] J. C. Pettibone, "Testing the effect of time pressure on asymmetric dominance and compromise decoys in choice," Judgment \& Decision Making, vol. 7, no. 4, pp. 513-521, 2012.

[8] T. Connolly, J. Reb, and E. E. Kausel, "Regret salience and accountability in the decoy effect," Judgment \& Decision Making, vol. 8, no. 2, pp. 136-149, 2013.

[9] M. Lichters, P. Bengart, M. Sarstedt, and B. Vogt, "What really matters in attraction effect research: when choices have economic consequences," Marketing Letters, vol. 28, no. 1, pp. 1-12, 2017.

[10] J. S. Trueblood and J. C. Pettibone, "The phantom decoy effect in perceptual decision making," Journal of Behavioral Decision Making, vol. 30, no. 2, pp. 157-167, 2017.

[11] Q. Meng, Z. Li, J. Du, H. Liu, and X. Ding, "Negotiation for time optimization in construction projects with competitive and social welfare preferences," Complexity, vol. 2019, Article ID 3269025, 13 pages, 2019.

[12] Z. Li, X. Lv, H. Zhu, and Z. Sheng, "Analysis of complexity of unsafe behavior in construction teams and a multiagent simulation," Complexity, vol. 2018, Article ID 6568719, 15 pages, 2018.

[13] Q. Meng, J. Chen, and K. Qian, "The complexity and simulation of revenue sharing negotiation based on construction stakeholders," Complexity, vol. 2018, Article ID 5698170, 11 pages, 2018.

[14] M. F. Shams and H. Kordlouie, "Studying the behavior of fund managers at Tehran stock exchange," African Journal of Business Management, vol. 6, no. 11, 2012.

[15] X. Xu, Q. Li, L. Peng, T. L. Hsia, C. J. Huang, and J. H. Wu, "The impact of informational incentives and social influence on consumer behavior during Alibaba's online shopping carnival," Computers in Human Behavior, vol. 76, pp. 245254, 2017.

[16] T. Zhang and D. Zhang, "Agent-based simulation of consumer purchase decision-making and the decoy effect," Journal of Business Research, vol. 60, no. 8, pp. 912-922, 2007.

[17] A. K. Banerjee and P. C. Padhan, "Herding behavior in futures market: an empirical analysis from India," Theoretical Economics Letters, vol. 7, no. 4, pp. 1015-1028, 2017.

[18] M. E. Schramm, K. J. Trainor, M. Shanker, and M. Y. Hu, "An agent-based diffusion model with consumer and brand agents," Decision Support Systems, vol. 50, no. 1, pp. 234-242, 2010.

[19] Y. Shi and J. C. Guan, "Small-world network effects on innovation: evidences from nanotechnology patenting," Journal of Nanoparticle Research, vol. 18, no. 11, p. 329, 2016. 
[20] Q. Meng, Z. Li, H. Liu, and J. Chen, “Agent-based simulation of competitive performance for supply chains based on combined contracts," International Journal Of Production Economics, vol. 193, pp. 663-676, 2017.

[21] B. Bhole and B. G. Hanna, "Word-of-mouth communication and demand for products with different quality levels," Computational Economics, vol. 46, no. 4, pp. 627-651, 2015.

[22] J. D. Bohlmann, R. J. Calantone, and M. Zhao, "The effects of market network heterogeneity on innovation diffusion: an agent-based modeling approach," Journal of Product Innovation Management, vol. 27, no. 5, pp. 741-760, 2010.

[23] K. Lee, H. Lee, and C. O. Kim, "Pricing and timing strategies for new product using agent-based simulation of behavioural consumers," Journal of Artificial Societies \& Social Simulation, vol. 17, no. 2, p. 1, 2014.

[24] W. M. Hedgcock, R. S. Rao, and H. Chen, "Choosing to choose: the effects of decoys and prior choice on deferral," Management Science, vol. 62, no. 10, pp. 2952-2976, 2016. 